\title{
Review of Comprehensive Assessment of Power Transformer
}

\author{
Cuiling Zhang, ${ }^{1, *}$, Yi Ning ${ }^{2}$ and Yahe Shi ${ }^{2}$ \\ ${ }^{1}$ Ningxia Institute of Science and Technology, Shizuishan Ningxia 753000, China \\ ${ }^{2}$ School of Information Science \& Engineering, Northeastern University, Shenyang Liaoning 110819, China \\ ${ }^{*}$ Corresponding author
}

\begin{abstract}
Since the power transformer is an important kind of electric power equipment in power system, in this paper, comprehensive evaluation of power transformer are divided into three parts: power transformer operation status, fault diagnosis and repair strategies. Furthermore, the evaluation methods of comprehensive evaluation analysis are proposed, which means, on-line monitoring, the method of fault diagnosis and condition-based maintenance strategy decision-making method. Summarizing the research thought, the application conditions and the research progress of these methods. Finally, some open problems for comprehensive evaluation of power transformer in this area are introduced.
\end{abstract}

Keywords-power transformer; operating status; comprehensive assessment; condition-based maintenance strategy; decision-making method

\section{INTRODUCTION}

The safety of power system includes the safety of power devices and power system operation ${ }^{[1]}$. Individual fault of main devices such as transformer and breaker may seriously affect the safe operation of whole power system. Rapid development of national power industry will cause the reducing schedule happen occasionally, and the setup and debug of equipment may fail to execute seriously according to the operating instruction, which will be the Trojan horse later.

The primary equipment faults over $220 \mathrm{kV}$ in operation are shown in table 1 according to document ${ }^{[2]}$.

TABLE I. T2009 ANNUAL NATIONAL ELECTRIC POWER EQUIPMENT OPERATION FAULT STATISTICS (FREQUENCY)

\begin{tabular}{|c|c|c|c|c|c|c|}
\hline $\begin{array}{l}\text { No } \\
\text {. }\end{array}$ & $\begin{array}{c}\text { Voltage } \\
\text { Grade }\end{array}$ & line & bus & $\begin{array}{l}\text { transform } \\
\text { er }\end{array}$ & $\begin{array}{l}\text { generator } \\
\text { over } \\
100 \mathrm{MW}\end{array}$ & $\begin{array}{l}\text { capacit } \\
\text { or }\end{array}$ \\
\hline 1 & $220 \mathrm{KV}$ & 1354 & 28 & 47 & \multirow{5}{*}{$\begin{array}{l}13 \text { faults } \\
113 \\
\text { abnormal } \\
14 \text { external }\end{array}$} & - \\
\hline 2 & $330 \mathrm{KV}$ & 87 & 0 & 1 & & - \\
\hline 3 & $500 \mathrm{KV}$ & 363 & 5 & 13 & & - \\
\hline 4 & $750 \mathrm{KV}$ & 4 & 0 & 0 & & - \\
\hline 5 & $1000 \mathrm{KV}$ & 0 & - & 1 & & - \\
\hline 6 & $\begin{array}{l}\text { DC } \\
\text { transmissi }\end{array}$ & 25 & 1 & - & - & 47 \\
\hline Total & & 1833 & 34 & 62 & 140 & 47 \\
\hline
\end{tabular}

From table one can figure out that the probability of transformer fault is on the third place of the whole power system. Therefore, as a key and main device in the system, power transformer's operating condition directly influence the quality of power system, and its safety and reliability will directly influence the operating cost and economic effectiveness. Proceeding real-time monitoring and fault predication and diagnosing transformer status are very important works; Finding power transformer (PT for short) fault and keeping it in higher maintaining level in operation process are most significant to the safety operation of power system.

Nowadays in domestic and overseas, the hot topics in PT status evaluation research include the operating state evaluation, fault diagnosis and fault prediction to $\mathrm{PT}^{[3]}$. Comprehensive judgment to PT can be divided into three aspects: operating status, fault diagnosis and status maintenance strategy, and they are the main research direction to PT at present. Figure 1 shows the comprehensive judgment analysis structure of PT.

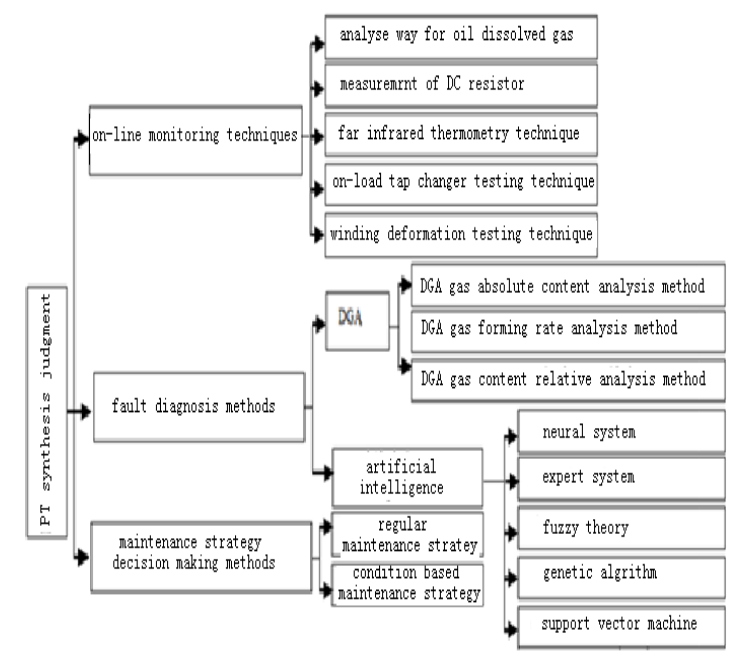

FIGURE I. THE FIGURE OF COMPREHENSIVE ASSESSMENT CONTENT STRUCTURE OF POWER TRANSFORMER

The main fault diagnosis method to PT is to make coding diagnosis (which is called IEC 3 ratios) based on the 3 ratios of dissolved gas in transformer oil proposed by the International Electric Technical Commission (IEC for short), by which the successful rate of fault diagnosis can be $80 \%$ or more ${ }^{[4]}$. To make on-line test to PT is very important to PT and whole grid safe operation. It is needed to know the real insulation status in transformer in time, to fine the hidden danger in inner PT as soon as possible, and to block the development and enlargement of hidden fault of transformer, so as to wipe out 
the fault when it is in its beginning. On the other hand, a decision can be made that if the service is needed (including the maintenance strategy used) and other treatment in case of damage. Hence this work has very important effect to enhance the reliability of power system ${ }^{[5]}$.

\section{ON-Line MONITORING TECHNIQUE OF POWER TRANSFORMER}

The main method of traditional detection means for PT are: preventive test detection, on-line monitoring, routine touring detection and fault synthetic judgment, etc. [6]. Many monitoring targets are included, such as dissolved gas analysis(DGA), pressure-tight test of insulation oil, DC resistor and insulation resistor of winding, winding deformation test, absorption ratio test, polarization index test, insulating dielectric loss angle tangent monitoring, insulator pressure-tight test, far infrared thermometry monitoring, on-load tap changer characteristics test and low voltage short circuit impedance test[6].

\section{The Technique of Oil Dissolved Gas Analysis}

There are many ways to make fault diagnosis to PT. The effective means in status detection technique is transformer DGA analysis, which has been widely used because the latent inner fault can be found early ${ }^{[7]}$. Statistics shows that over $50 \%$ transformer fault in power system are detected by DGA analysis result ${ }^{[8]}$. On-line oil dissolved gas technique is carried out depending on different gases such as $\mathrm{H}_{2}, \mathrm{CH}_{4}, \mathrm{C}_{2} \mathrm{H}_{6}, \mathrm{C}_{2} \mathrm{H}_{4}$, $\mathrm{C}_{2} \mathrm{H}_{2}$, CO and $\mathrm{CO}_{2}$ when transformer fault occur, so the qualitative and quantitative detection can be fulfilled. The main detection means are hue chromatography, infrared chromatography, photo- acoustic spectrometry and sensor array ${ }^{[9]}$.

(1)Hue chromatography on-line detecting technique includes two key technologies named as oil-gas separation and gas detection, in which the mostly used way for oil-gas separation is polymeric membrane, like C201-6 on-line chromatography system by Canadian GAJI group and the research made by Wang-youyuan in Chong-Qing university; Gas detector mainly detects one-component (mainly $\mathrm{H}_{2}$ ) and multi-component quantity of gas in oil. In one-component detection, palladium grid FET and catalytic oxidation inductor as well as electrochemistry $\mathrm{H}_{2}$ inductor are mostly used [10], while the main product is made by Canadian SYPROTEC company, HYDRAN; in multi-component detection, the mainly useful means are thermal conductance sensor, hydrogen flame ionization sensor and semiconductor sensor, etc. ${ }^{[11]}$. The TRUE Gas on-line monitoring device made by American company AVO can detect gases as many as 8 , and it is the most effective instrument so far.

(2)Infrared spectrum is mainly used to directly determine gas type and content by spectrum analysis. Its characteristics are fast, high precision rate, non-contact, easy to be maintained, and various gases can be detected, but it is not sensitive to $\mathrm{H}_{2}$ and is too expensive; photo-acoustic spectrometry is a kind of detecting technique based on photo-acoustic effect ${ }^{[12]}$, and it detect the ability of gas absorbing optical energy in the optical-acoustic cabin ${ }^{[13]}$. $\mathrm{H}_{2}$ can be detected and is strong in anti-interference.
(3)Sensor array is to combine multi-gas sensor together, it can detect different components in mixed gas ${ }^{[14]}$, improving the detection efficiency and sensitivity of gas dissolved in oil.

\section{Measuring Technique of DC Resistance}

Detecting DC resistance of PT winding is an important parameter in detecting a transformer. In comprehensive judgment to transformer winding fault (including the connection of lead and wire, branch switch and winding, and so on), DC resistance value and its imbalance provides important information. Mr. Guo-yu and others proceeded extensive research on DC resistance on-line measurement, as shown in document ${ }^{[15]}$. By means of DC resistance test, various cases can be checked, such as soldering situation of winding contact, if the short circuit between turn-to-turn exist, if the voltage branch point connecting well, if the real position is the same as indicated, the wire condition, if there exist breaks case in duplex winding ${ }^{[15]}$.

There are two ways to measure DC resistance: common method and fast measuring. Common method includes voltage-ampere based on ohm law, balanced bridge (single arm and double arms) based on Wheatstone bridge as well as DC resistance tester ${ }^{[16]}$.

Fast measuring based on some key techniques like wider measuring range, higher accuracy, faster speed, little influence by winding inductance, automatically exchange winding group and treat the result, including static fast measuring such as resistance mutation, bridge mutation, flux pump, short circuit degauss, magnetism assisting, and randomly measuring, two step oscillating and dynamic fast measuring based on identical theory, etc. ${ }^{[17]}$.

\section{A. Infrared Thermometric Techniques}

There are many advantages for far infrared thermometric technique : non-contact, wide measuring range, high measuring accuracy, fast speed, high sensitivity and long service life, and transformer cease running and dismount is unnecessary, so it is wildly used in inner fault detection to $\mathrm{PT}^{[18]}$.

Since 60's for overseas and 70's for domestic in last century, thermal imager began to be used in power device fault diagnosis. Recently rich experiences have been accumulated on detecting inner fault of conducting loop and magnetism loop of large power devices, not only the fault type can be detected, but also the location and its severity can be measured quantitatively ${ }^{[19]}$.

\section{B. Testing Technique of OLTC Characteristic}

By measuring the on-load tap changer's switching time, cycle and switching wave form, whether the performance of OLTC of PT is good or not can be grasped. The fault type of OLTC mainly includes: switch loose (or to say, interlock), switchover failed or switching too slow and abort, selector switch mismatch with option switch, OLTC seal leak, electric mechanism fault and parts deformation fault, switch point contact unhealthy, switch discharge, shaft broken, switch insulation damp, switching limit excessive, contact system problem, and so on. Therefore, mechanism performance test to 
the OLTC could forecast the probability of mechanism fault and estimate the fault type, hence to ensure the operation safety of power system ${ }^{[20]}$.

\section{Testing Technique of Winding Deformation}

By means of testing the winding deformation, one can know that if the transformer winding deformation or shifting occur after short circuit. The main methods of winding deformation are: frequency response, short circuit impedance, low voltage impulse, on-line monitoring leakage reactance, capacitance variation, ultrasonic detection, vibrating monitoring, off-line and on-line methods could detect and decide if the winding deformation and shift occur when short circuit fault happens ${ }^{[21]}$.

\section{The Fault Diagnosis Method of Power TRANSFORMER}

There are two ways to make fault diagnosis and status estimate to PT: oil dissolved gas method (DGA) based on oil chromatographic analysis and artificial intelligence based on smart control theory (witch can be called analysis method based on rule and intelligence).

\section{A. The Fault Diagnosis Method Based on DGA}

Presently the fault diagnosis based on DGA is widely used in transformer fault diagnosis ${ }^{[22]}$, which is based on the Hastead hypothesis. The hypothesis mentioned to carry on thermodynamic analysis for the course of gas status hydrocarbon compounds, considering that for balanced pressure under different temperature the proportion between one hydrocarbon gas and the other depends on hot spot temperature, accordingly Hastead hypothesis established: assumes the separating rate of hydrocarbon gas vary with temperature, while every gas achieved its maximum separating rate on different temperature respectively, then the relative separating rate under special temperature are fixed. Depending on this hypothesis, along with the temperature goes higher, the sequence of maximum separating rate of oil dissolved gas would be: $\mathrm{H}_{2}, \mathrm{CH}_{4}, \mathrm{C}_{2} \mathrm{H}_{6}, \mathrm{C}_{2} \mathrm{H}_{4}, \mathrm{C}_{2} \mathrm{H}_{2}$. On this basis, various scholars enrich and develop the fault diagnosis based on DGA: Donable proposed two ratio method to distinguish thermal fault from electric fault; Davis presented oxy-hydrogencarbon triangle map discrimination; Dewey put forward a triangular chart based on $\mathrm{CH}_{4}, \mathrm{C}_{2} \mathrm{H}_{4}, \mathrm{C}_{2} \mathrm{H}_{2}$ relative amount; Rogers successively raised three ratio and four ratio method; electric-aided research method was figured out in Japan; China proposed the improved electric-aided research method. All these methods are widely used in real work ${ }^{[7]}$.

Generally the fault analysis ways using DGA can be divided into three classes: the first is on the basis of the absolute value of oil dissolved gas; the second depends on the occurring rate of oil dissolved gas amount; the third is judged by calculating relative proportion of different oil dissolved gas [23].

Method that based on absolute value of oil dissolved gas is according to analyzing limited value of oil dissolved gas; method that based on occurring rate of oil dissolved gas is depending on the limited value of relative and absolute rate of gas production; method that fault diagnosis to PT based on relative ratio of oil dissolved gas includes coding and nonecoding. International Electro-technical Commission (IEC) uses three ratio method to make coding diagnosis, and improved three ratio method is used in China.

Lan-shuli and others made analysis and judgment to PT fault type by absolute value of oil dissolved gas, occurring rate of oil dissolved gas, three ratio method, and the examples verified that the method above is correct and feasible $\mathrm{e}^{[23]}$.

Yang-tingfang and others extended DGA analysis method by introducing BORDA method and increased the accuracy of fault diagnosis ${ }^{[24]}$.

There are some disadvantages for three ratio method such as "lack of coding, coding rule boundary absolution, fault classification vague". For this reason, recently artificial intelligent methods including expert system, neural network, fuzzy theory and genetic algorithm are used in PT fault analysis, some better results have been obtained. This is a new way to analyze the PT fault ${ }^{[25]}$.

\section{B. Artificial Intelligent Diagnosis Method}

\section{1) The fault diagnosis method based on ANN}

Artificial Neural Network (ANN for short) has inherent study ability, generalization ability, self-adaptation ability and linear mapping ability. It is a system handling information that simulate human brain structure and cognitive process, having powerful self-study and data handling capacity and reflecting highly nonlinear input-output relation. Since first proposed in 1943 , it has been rapidly developed and is called to be an important branch of artificial intelligence. It could archive parallel distributed processing, self-adaptation, associative memory, so a new research approach was opened up in intelligent fault analysis area ${ }^{[26]}$.

According to the feature of transformer fault diagnosis, an artificial neural network method for PT fault diagnosis was put forward and researched ${ }^{[27]}$. A neural network model was established using partitioning technique and made the input and output corresponding to fault symptom and type respectively. A transformer fault diagnosis method was put forward based on BP (backward propagation) neural network $^{[28]}$. It took 6 gases in insulating oil as input of neural network, established a BP neural network model for diagnosing to 7 faults including transformer imbecility discharging, high energy discharging, partly discharging, low temperature overheating, mesotherm overheating, high temperature overheating, mixed fault high overheating and high energy discharging. The model established is simple and convenient, performance good, and accuracy higher. Aiming at disadvantages of BP algorithm such as seeking rate of convergence low, having local minimum in seeking space and easy to cause vibration, an improved wavelet neural network diagnosis algorithm using changing learning rate and momentum was proposed to analysis oil dissolved gas ${ }^{[29]}$. A fault diagnosis method based on quantum neural network information fusion was presented aiming at the feature of various fault types and unidentified fault information, large data volume and none-regularity ${ }^{[30]}$. It brought multi-PT fault parameter information into quantum neural network to make 
local fusing diagnosis, then brought them into decision fusion network to make global fusion, finally 5 PT faults were diagnosed as well as reliability evaluation was given. In document ${ }^{[31]}$, PNN probability network method was used to diagnose the PT fault by analyzing the structure and principle of PNN probability network, aiming at powerful none-linear sorting ability of probability neural network model. The training time for PNN is less than that of BP and its prediction accuracy is higher, as well as its higher generalization ability. These advantages make the PNN network be effectively used in transformer fault diagnosis with certain operability. Various wave-net neural network models were proposed to enhance the fast learning ability of neural network ${ }^{[32]}$.

\section{2) The fault diagnosis method based on expert system}

Expert system (ES) is one of the most active and most extensive artificial intelligent techniques, with vast expert level knowledge and experiences in some area. It estimates and judges by means of knowledge and experiences offered by one or more experts from some area and then forming intelligent computer program system, simulating human expert's decision-making process to solve complex problems ${ }^{\text {[3] }}$. For those problems that mathematic model is hard to be established or expert knowledge and experiences are relied on, the expert system has its own superiority ${ }^{[34]}$.

A fault diagnosis expert system to transformer was proposed. Transformer fault phenomenon, DGA analysis result and electric test data were taken as a piece of information, with which the characteristic gas, three ratios, fault phenomenon and electric test data were used to diagnose the transformer fault alone or comprehensively ${ }^{[35]}$. Forward reasoning and $\mathrm{C}$ language were used so the operation is convenient and expert knowledge is abundant, fitting the professionals to use. It was proposed to solve the transformer fault diagnosis using rough set formed by expert knowledge base due to the problem of that hard to gain complete knowledge ${ }^{[36]}$. Using historic fault data to form a simple decision table, calculating the regular rough membership, finally the node network rule set was formed to meet the needs of confidence coefficient of different simplifying level. A fault diagnosis method based on connecting confidence rule base with DGA was proposed, which has a powerful practical value [37]. A PT insulation fault diagnosis expert system that developed taking DGA as characteristic quantity was described, with which the transformer insulation condition could be comprehensively diagnosed, the fault type was correctly recognized, and the fault rough location, the order of severity, and its development tendency could be provided ${ }^{[38]}$. A PT fault diagnosis expert system based on knowledge base as a kernel was raised because of characteristic of multiple fault mechanism [39]. Aiming at the feature of large amount of knowledge in DGA base, rule confidence coefficient was used to make conflict reduction to fault information matching condition. According to the probability feature of PT fault type, a dynamic knowledge base model was established to store recent fault. The dynamic knowledge base was scanned by inference engine, hence each data table of data base was built, and the inference efficiency was increased by using major key and external key. An extendable fault diagnosis expert system for oil dissolved gas was delivered, by integrating fault decision rule gained from traditional three ratios, statistics learning method and practical experience, thus an expert system that could add new rule was created ${ }^{[40]}$.

The disadvantage for expert system theory to make fault diagnosis is that the scientific and accurate knowledge base decides the hit ratio of fault diagnosis.

\section{3) The fault diagnosis method based on fuzzy theory}

Fuzzy theory (FT) has a comparatively powerful structural knowledge presentation skill. It could mainly simulate logic thinking of human brain, and could change the uncertain or certain knowledge into fixed quantity. In fault diagnosing, the relation between fault and symptom may be fuzzy, and diagnosing result may be fuzzy because of inaccurate concept description, so the fundamental thought is to establish a fuzzy relation matrix between fault symptom and fault causes using expert experience $^{[41]}$.

Aiming at the insufficiency of PT fault diagnosis, fuzzy clustering analysis theory, method and distinguishing principle of clustering effect were introduced, using which to make fault diagnosis to PT ${ }^{[42]}$. Dissimilarity thought was utilized, samples were treated in weighted approach, algorithmic initial membership was set reasonably, traditional coding missing was made up, so its convergence is very good ${ }^{[43]}$. Utilizing complement each other's advantages of fuzzy sets and decision-making tree fusion, the disadvantages of fuzzy sets and decision-making tree could be overcome respectively, thus the accuracy and efficiency of transformer fault diagnosis could be enhanced ${ }^{[44]}$. In order to avoid the unification of membership function, a fault probability diagnosis based on fuzzy membership function transformer experimental data was presented, in which the assigning method was used to select the membership function [45]. A distributed MAS fault diagnosis system structure was employed to design a transformer fault diagnosis model combining fuzzy information fusion method [46]. This structure overcame disadvantages such as weak of instantaneity and reliability. Document [47] proposed a new transformer fault diagnosis comprehensive model using fuzzy theory, probability reasoning and economic cover sets depending on electric test data, DGA analysis and observed information. The historic service records were fully considered, the expert's knowledge were furthest integrated, the cause-result and strength relation between fault nature and fault symptom was built, so the diagnosis conclusion could be more accurate and more reliable. Based on fuzzy multi-attribute decision-making theory, a suggestion was proposed merging the symptom and test data, complementing each other's advantage to various kind of information, and diagnosing the symptom phenomenon ${ }^{[48]}$. It diagnoses the preventive test using fuzzy probability model, and fuses the diagnosis result by D-S evidence theory.

The existing problem based on fuzzy theory to diagnose transformer fault is that the method to decide membership function of fuzzy relation matrix is relatively simple, so the convincible objective gist is insufficient.

4) The fault diagnosis method based on genetic algorithm

Genetic algorithm is searching optimizing on computer to 
simulate biological evolution mechanism based on natural selection and genetic mechanism. It makes self-adapting searching in huge space in order to find the optimal or quasi-optimal solution, with stronger practicability and robustness. First, every chromosome generated from algorithm was evaluated, then chromosome was changed by fitness, with the purpose of increasing the breeding chance to high fitness chromosome ${ }^{[49]}$.

A chromosome multi-crossover genetic algorithm was put forward, a neural network was designed that automatically adjust network parameter connection weight and bias, a transformer fault diagnosis system was built that integrating DGA, genetic algorithm and neural network together ${ }^{[50]}$. It has a certain accuracy to make fault diagnosis such as transformer overheating, discharging and damp affecting. Optimized fuzzy rule base was used in genetic algorithm, bootstrap was adopted to make processor expansion for data examples, so the precision and accuracy were enhanced because different fault type has the same sample size ${ }^{[51]}$. In order to evaluate transformer fault state, a method combining genetic algorithm and supporting vector machine was used, in which the optimum memory antibody sets of fault type was trained by gray artificial immune algorithm, 5 adjacent comprehensive decision method was used to sub-classify the fault type depending on gained optimum memory antibody sets, single and multi-fault sorting were fulfilled and fault location delivered ${ }^{[52]}$. In order to quickly enlarge the searching space, stabilizing individual multi-formity in group, effectively enhance global searching ability and rate of convergence, a parameter searching optimizing method based on improved genetic algorithm (IGA) was proposed, which could be used in predicting fault gas in transformer oil ${ }^{[53]}$.

How to establish a reasonable fault diagnosis model is a main "bottleneck" in using genetic algorithm theory to make fault diagnosis.

\section{5) The fault diagnosis method based on SVM}

Support vector machine (SVM) belongs to a small sample learning method, very good generalization ability could be taken when training sample is smaller. Now some researchers applied it to transformer fault diagnosis ${ }^{[54]}$. A method was introduced that using support vector machine to establish PT fault diagnosis hierarchical decision making model, in which the transformer fault type diagnosis was done by distributive characteristics of typical fault gas quantity in higher dimensional space ${ }^{[55]}$. A multilevel support vector machine was described, constructing minimum classifiers to make better space zone eliminated the none-classified space ${ }^{[56]}$. This method resolves specially to 2 classifiers and could be further extended with better generalization ability. A M-ary support vector machine was used to realize transformer fault diagnosis [57].

The key to success for support vector machine is the selection of parameters in model. Right now support vector machine theory works by mapping the kernel function from low-dimensional space to high-dimensional space, thus it is hard to be explained or understood as well as the calculation cost is higher when common grid searching is used.

\section{Maintenance Method of POWER Transformer}

The PT device maintenance strategy experienced a long term time based maintenance stage ${ }^{[58]}$. This strategy was based on determinate schedule, causing two extremes as "no maintenance when needed" and "maintenance excess". This will cause maintenance excess, cost increase and low reliability. Long term experience shows that the probability of PT fault presented a "tub" curve feature with time: high on both sides, and low in middle. In PT initial operating stage the fault probability is higher (initial failure), and would be reduced and maintains in a low level (fault is infrequency) along with time increased. When it runs a certain times, the fault probability shows a rapid increasing trend again (consume fault). The maintenance strategy should vary with the corresponding variation of PT device fault probability. Therefore, condition based maintenance ${ }^{[59]}$ (CBM, or to say, reliability-centered maintenance, RCM) was born at the right moment. Condition based maintenance is a reliable, cost reasonable strategy mainly based on reliability, safety, on-line state measuring data and historical data to formulate maintenance plan.

Power transformer condition-based maintenance decision models experienced four stages named as original stage, initial stage, system stage and perfect stage respectively ${ }^{[1]}$. During these four stages, from the beginning of basic transformer status evaluation assisted by original data accumulation coming from periodic maintenance, four stages were experienced: periodic maintenance is master and condition based maintenance is slave (original stage), attach equal importance to periodic maintenance and condition based maintenance (initial stage), periodic maintenance is slave and condition maintenance is master (system stage), condition base maintenance decision (perfect stage). The development of maintenance decision model experienced data historic accumulation, off-line data processing, on-line monitoring real-time data processing, and finally the expert system data-base was formed. The ultimate goal and development direction of PT maintenance decision research would growing with the development of on-line monitoring technology, while right now domestic and overseas research target are all focus on PT condition based maintenance ${ }^{[60]}$.

Maintenance decision model regarding to uncertainty goal was proposed in order to solve the uncertainty factor problem which would influence maintenance strategy ${ }^{[61]}$. The uncertainty of transformer characteristic quantity could be effectively settled by establishing indicator system of reflecting transformer insulation status and by dividing system status using connection degree and set pair analysis theory; however, if different characteristic quantity result are directly be weighted, then it will be wrong when bigger difference exists in different characteristic quantity evaluation results.

A transformer status evaluation strategy was put forward based on fuzzy theory ${ }^{[62]}$. Half-drop trapezoid model and fuzzy comprehensive evaluation were used in this method to determine transformer operation status through changing and assessing various transformer status parameters, and then the maintenance strategy could be worked out according to the order of evaluation. 
On the basis of multi-attribute and multi-stage of decision aim, scholar YUAN Zhi-jian, SUN Xin-cai etc. used fuzzy multi-attribute group decision and compromise group decision to discuss the application of fuzzy multi-attribute group decision in transformer condition maintenance decision [63]. Later they utilized simple fuzzy weight averaging decision and fuzzy compromising decision to contrast, discussing the practicability of fuzzy multi-attribute decision method in PT condition based maintenance. Scholar ZHANG Yi-yi etc. constructed a condition based maintenance strategy optimized model which could overall considers transformer running reliability and economy aiming at multi-factors such as the reliability, economy and healthy status in transformer condition based maintenance ${ }^{[60]}$. By seeking the relation between transformer healthy state and fault rate, using particle swarm method to optimize maintenance decision for constructed model, aiming at to find an optimal maintenance decision result compositing reliability with economy.

PT condition based maintenance is a decision course which the feature of random, multi-attribute target coexisted and influence factors are fuzzy. Therefore, although some results have been gained in determining transformer condition based maintenance using every method alone, but the ideal way to establish a suitable PT condition based maintenance decision model is to comprehensively utilize various decision methods. The research emphasis is device reliability analysis, but because of complicated fault structure and evaluation indication variety and on-line detect technique's immaturity, the condition maintenance development is limited, so new theories and methods is badly needed to be introduced for PT maintenance.

\section{RESEARCh TENDENCy OF POWER Transformer COMPREHENSIVE EVALUATION METHOD}

\section{A. The Issue in Power Transformer Comprehensive \\ Evaluation}

The template is designed so that author affiliations are not repeated each time for multiple authors of the same affiliation. Please keep your affiliations as succinct as possible (for example, do not differentiate among departments of the same organization). This template was designed for two affiliations. During the research process of PT fault diagnosis, judgment based on DGA is still the main information source for researching PT condition maintenance and fault diagnosis. Traditional methods and artificial intelligent means exist prominent drawbacks in fault diagnosis and status analysis.

a)During transformer fault detection there exists such problems as technique immaturity, lower fault location and date detect accuracy, so it is easy to cause misdiagnosis and missing diagnosis ${ }^{[57]}$;

b)The accuracy and speed of Intelligent diagnosis and evaluation established on DGA data is lower due to insufficient characteristic parameter indication of limited bearing capacity of PT short circuit and representing state level [60];

c)Expert system is based on knowledge rule and simulating expert in experiential knowledge to fulfill fault diagnosis and maintenance strategy, so the "bottle neck" problem for expert system to make accurate fault diagnosis is the integrity of judging rule and knowledge acquisition ${ }^{\text {[64]; }}$;

d)The convergence rate of artificial neural network is slow, a prior experience is needed for its net structure and parameter setting, a very important issue is how to gain optimum network structure of special problem ${ }^{[65]}$.

e)Fuzzy theory method takes subjective factors as a master when fuzzy membership function is determined. The method is comparatively simple and objective pursuant credibility is scarce; the correct diagnosing premise of fuzzy comprehensive evaluation is to build a fuzzy relation matrix conforming to real; it is somehow difficult to establish a fuzzy relation between fault cause and fault symptom ${ }^{[66]}$;

f) When genetic algorithm is used in PT condition evaluation, there exists a problem that the evaluation quantity of status is incompletely considered, so there is a deserved researching question that how to set up a proper evaluation model.

\section{B. Development Tendency of Power Transformer Comprehensive Evaluation Research}

Recently the research work on PT fault diagnosis and condition based maintenance decision model is developing towards the multi-methods mixed, so as to enhance the accuracy of diagnosis and decision ${ }^{[67]}$. Now the development tendency of transformer fault diagnosis are fault information multiple-dimensioning, diagnosis theory diversification and diagnosing technique intelligence. Their specific behaviors are as follows:

1) The research for data support based on multidimensional information

Right now running data obtaining method in power transformer operation appears to diversified situation along with the development of electrical technology. Monitoring and detecting techniques, the quantity and species of information obtained is substantially increased. The information includes electrical measuring data, gaseous phase measuring data, mechanical vibration data, tour data, historic operation data and products family defect, etc. The status analysis technique using decision method utilizes only one or several kinds of feature information of the above, so the diagnosis result or evaluation level would be unreliable, the reflected status are incomplete and uncertain. Thus in future the issue needs to be solved in transformer maintenance and fault diagnosis would be obtaining, processing and utilizing information in many ways.

2) The research of combining various intelligent method

Various intelligent methods were used in making decision for PT fault diagnosis and condition based maintenance strategy, each having its own characteristics. They are different in data information processing manner, logical inference mechanism and expressing respect, describing different logical relation and mechanism feature from different sides of the problem. Yet these descriptions could not fully reflect the complex relations between fault symptoms and their causes, 
having different expression conclusions and accuracies, and a certain one-sidedness and limitation exists. Therefore, how to comprehensively utilize advantages of various intelligent ways and how to chose intelligent methods reasonably for treating information would be an important research direction for combining intelligent methods in order to reinforce PT fault diagnosis and condition based maintenance strategy.

3) The research of diagnosis and decision method when information missing

In the case of information missing or information inaccuracy, the misjudgment rate is higher to existing fault diagnosis and condition base maintenance. There are many reasons to cause information missing, including transformer test items incomplete, fault information missing, inaccurate data measuring, etc. For this reason, at present the issue to be solved in transformer status evaluation is how to process missing information and how to enhance judgment accuracy.

4) The research on fault location of power transformer

Recently most comprehensive evaluation and maintenance strategy of PT operation status is still based on DGA data diagnosis to fault nature and location. Due to the complex transformer structure and a wide range of fault types, the research and application are still in its exploration stage. Though there is tentative diagnosis to combine other electrical test, while they are limited only for several special fault types, without universal meaning. It is a worthy research issue that how to combine transformer structure feature, fault occurring mechanism together to estimate fault location, and directly describe them so as to guide formulating maintenance strategy [1].

\section{ACKNOWLEDGMENT}

This article is supported by the construction projects of the key disciplines of electrical engineering and automation of the Ningxia Education Department

\section{REFERENCES}

[1] SUN Cai-xin, "Present situation and development of condition on-line monitoring and diagnosis technology for power transmission and transformation equipment,” Electric Power, vol. 38(2), pp.1-6,2005.

[2] SHEN Xiao-fan, SHU Zhi-huai, LIU Yu, et al, "Operation situation of protective relaying of State Grid Corporation of China in 2009”,Power System Technology, vol. 35(2) pp. 189-193, 2011.

[3] ZHANG Yi-yi, "Study on Life Cycle Cost based Maintenance Decision Making for Power Transformers Considering Condition Assessment and Insulation Life Assessment”, Chongqing: Chongqing University, June 2014.

[4] SUN Cai-xin, CHEN Wei-gen, LI Jian, "Electrical equipment in oil gas on-line monitoring and fault diagnosis technology", Beijing: Science press, 2003.

[5] CHEN Wei-gen , Sun Cai-xin , "Study on fault diagnosis methods of transformer DGA with fuzzy model hierarchy classification ”, ICMEP, vol.(09),pp. 167-171,2000.

[6] China preventive test code for electric power equipment (DL/T597-1996), Beijing : Electric power press,1997.

[7] SUN Hui, LI Wei-dong, SUN Qi-zhong, "Electric power transformer fault diagnosis using decision tree ", Proceedings of the CSEE, vol. 21(2),pp.50-55,2001.

[8] China Power Equipment Co.500KV transformer quality compilation, Beijing: Ministry of electric power research institute, 1996.
[9] DengHua Mei, HuaQing Min, “A Fuzzy Information Optimization Processing Technique for Monitoring the Transformer in Neural-Network On-line ”, Proceedings of 2005 IEEE International Conference on Dielectric Liquids, Cambria, Portugal,pp.273-275 ,2005.

[10] Lin Jin-po, Zhao Ji-yin, Zheng Rui-rui, Liu Yu, “Fault Diagnosis System of Transformer Based on Gas Chromatography ”, Proceedings of the Fifth International Conference on Machine Learning and Cybernetics, Dalian China , pp.809-813, 2006.

[11] Lzzularab M A, Aly G E M., Mansour D A, “On-line Diagnosis of Incipient Faults and Cellulose Degradation Based on Artificial Intelligence Methods ” 2004 International Conference on Solid Dielectric , Toulouse, France,pp.5-9,2004.

[12] ZHANG Chuan, WANG Fu, "Application of Photo- Acoustic Spectroscopy Technology to Dissolved Gas Analysis in Oil of Oi-l Immersed Power Transformer”, High Voltage Engineering, vol. 31(2),pp.84-86,2005.

[13] ZHANG Hao-yang, CAI Zhi-yuan, ZHANG Jun-yang, “An Overview of On-line Monitoring Technology for Dissolved Gas Content in Transformer Oil”, Northeast Electric Power Technology, vol.8,pp.48-50,2006.

[14] YANG Qiping, Xue Wude, Lan zhida, “Application of Group Sensor for Transformer On-line Monitoring ”, 2005 IEEE /PES Transmission and Distribution Conference \& Exhibition, Dalian China, pp.1-4,2005.

[15] GUO Yu, "Online Measurement of Transformer Winding DC Resistance ”, North China Electric Power University, March 2012.

[16] SU Jin-fu, “Analysis on Measurement of Direct Current Resistance ”, Transformer, vol.42(8),pp. 22-26,2005.

[17] WANG Liang, “The study of transformer's DC resistance rapid measurement”, Beijing: North China Electric Power University, December 2001.

[18] CHEN Lin, TANG Zhong, CUI Hao-yang, "The Realization of Infrared Thermometric Techniques of Electric Equipment ", Electrical Measurement \& Instrumentation, vol. 50(568),pp.64-69,2013.

[19] ZHANG Han-jun, "Research of Infared Remote Monitoring for Power Equipmen t”, Beijing: North China Electric Power University, December 2008.

[20] ZHAO Tong, "Studies on On-line Monitoring and Fault Diagnostics for Mechanical Condition of On-load Tap Changers ”, Jinan: Shandong University, April 2008.

[21] Shu Naiqiu, zhou Can, Hu Fang, Liu QiSheng, Zheng Lingwei, “Study on ultrasonic measurement device for transformer winding deformation, ” Power System Technology, 2002, Proceedings, Power Con 2002, Kunming, China P.R.C., International Conference, IEEE CNF,Vol.3, pp. 1401-1404,2002.

[22] Guardado J L, Naredo J L, Moreno P, “A comparative study of neural network efficiency in power transformers diagnosis using dissolved gas analysis ” , IEEE Trans Power Del,vol.16(4),pp. 643-647,2001.

[23] AN Shu-li, SONG Kun, LIU Fang, “A Case Study on Fault Diagnosis of $500 \mathrm{kV}$ Transformer with Gas Chromatography Method”, High voltage apparatus,vol. 44(4),pp.381-382,2008.

[24] YANG Ting-fang, "Study on New Techniques of Online Monitoring and Fault Diagnosis for Power Transformer", Wuhan: Huazhong University of Science and Technology, April 2008.

[25] Su Q, Mi C, Lal L. L,et al. , “A fuzzy dissolved gas analysis method for the diagnosis of multiple incipient faults in a transformer”, IEEE Transactions on Power Systems,vol. 15(2),pp.593-598,2000.

[26] H Wu, X Li, "RMP Neural Network Based Dissolved Gas Analyzer for Fault Diagnostic of Oil-filled Electrical Equipment “, IEEE Transactions on Dielectrics and Electrical Insulation (S1070-9878), vol.18(2),pp. 495-498,2011.

[27] GUARDADO J L , NAREDO J L , MORENO P , et al., “ A comparative study of neural network efficiency in power transformers diagnosis using dissolved gas analysis”, IEEE Trans on Power Delivery, vol.16(4),pp. 643-647,2001.

[28] YU Jian-li, BIAN Shuai, “ Fault Diagnosis Model of Transformer Based on BP Neural Network”,Journal of System Simulation, vol.26(6), pp.1343 -1349,2014. 
[29] CHEN Wei-gen, PAN Chong, YUN Yu-xin, WANG You-yuan, SUN Cai-xin, " Fault diagnosticmethod for power transformer based on improved wavelet neural network algorithm”, Chinese Journal of Scientific Instrument, 8vol.29(7),pp.1489-1494,200.

[30] GONG Rui-kun, MA Liang, ZHAO Yan-jun, YANG Ping-ping, CHEN Lei, “ Fault diagnosis for power transformer based on quantum neural network information fusion”, Power System Protection and Control, vol.39 (23),pp.79-85,2011.

[31] WANG Gui-ying, ZHANG Shi-jun, PAN Si-yao, “ Investigation of Transformer Fault Diagnosis Method Based on PNN", Computer Measurement \& Control , vol.20(7),pp.1760-1762, 2012.

[32] ZHANG Q, BENVENISTE A, "Wavelet networks", IEEE Trans on Neural Networks, vol. 3 (6),pp. 889-898,1992.

[33] Cao Alin, Zhu Qinjun, Zhang Shengtao, et al., "BP neural network predictive model for Stray current density of a buried metallic pipeline”, Anti-corrosion Methods And Materials, vol.57(5),pp.234-237, 2010.

[34] Lin C E, Ling J M, Huang C L, “An expert system for transformer fault diagnosis using dissolved gas analysis”, IEEE Transactions on Power Delivery, vol.8(1),pp. 231-238,1993.

[35] MENG Da-wei, WEN Xiang-long, CHENG Jie-ying, ZHANG Ying-jian, DONG Yuan-gui, “A Fault Diagnosis Expert System for PowerTransformers", Heilongjiang Electric Power,vol.21(2),pp.33-37,1999.

[36] SHU Hong-chun, SUN Xiang-fei, SI Da-jun, “A RS approach to founding and maintaining ES knowledge base for fault diagnosis of power transformer" , Proceedings of the CSEE, vol. 22(2),pp.31-35,2002

[37] CHEN Jin-qiang, "Diagnosing Transformer Fault Based on RIMER Expert System and DGA”, High Voltage Apparatus, vol. 49(11),pp. 76-81,2013.

[38] CHEN Xin-gang, LI Tai-fu, "Research on Expert System of Transformer Diagnosis Based on DGA Characteristic Insulation Fault Parameters”, Transformer,vol. 42(1),pp.33-36,2005.

[39] LIU Shou-ming, HU Zhi-kun, WANG Mei-ling, "Expert System of Power Transformer Fault Diagnosis Based on Knowledge Base", Computer Measurement \& Control, vol.19(7),pp. 1569- 1572,2011.

[40] SHI Rui-feng, SHI Yong-feng, MU Jun, LI Hui, LIU Xiao-han, "Power Transformer Fault Diagnosis Expert System with Dissolved Gas Analysis in Oil”, Proceedings of the CSU-EPSA, vol. 26(12),pp.49-54,2014.

[41] Wu J D, Wang Y H, Bai M R, "Development of an expert System for fault diagnosis in scooter engine platform using fuzz-logic inference”, Expert Systems with Applications, vol.33(4),pp.1063-1075,2007.

[42] SONG Bin, YU Ping, LIAO Dong-mei, LUO Yun-bai, "Fuzzy Set Analysis of Dissolved Gas Applied to Fault Diagnosis of Power Transformer”, High Voltage Engineering, vol.27(3),pp.69-71,2011.

[43] DU Zheng-cong, NIU Gao-yuan, "Transformer Fault Diagnosis Based on Weighted Fuzzy Clustering Algorithm”, High Voltage Apparatus, vol.50(4),pp.42-48,2014.

[44] GU Kai-kai, GUO Jiang, “Transformer Fault Diagnosis Method Based on Compact Fusion of Fuzzy Set and Fault Tree”, High Voltage Engineering, vol.40(5),pp.1507-1513,2014.

[45] YUAN Lei, DU Lin, WU Jun-mei, AI Hua, “Transformer multi-parameter integrated fault diagnosis method based on fuzzy membership function”, high voltage apparatus, vol. 47(5),pp.35-42,2011.

[46] DING Tan, LI Meng, LIU Hui, “A Research on the Transformer Fault Diagnosis Based on the Fuzzy Information Integration”, Journal of Hubei University of Technology, vol.23(5),pp.21-23,2008.

[47] FU Yang, JIANG Yu-rong, CUI Chun-hong, CAO Jia-lin, "Fuzzy Theory and Probability Reasoning Applied for Identifying Power Transformer Fault,,High Voltage Engineering, vol.34(5),pp.1040-1044,2008.

[48] DU Lin, YUAN Lei, WANG You-yuan, "Power transformer fault fusion diagnosis using FMADM theory”, Journal of Chongqing University, vol.33(12),pp.1-7,2010.

[49] GOLDBERG D E, “Genetic Algorithms in Search, Optimization, and Machine Learning “. Addison-Wesley, 1989.
[50] DENG Hong-gui, LUO An, CAO Jian, DING Jia-feng, WANG Hui-hai, "Application of multi-point criss-cross genetic algorithm in transformer fault diagnosis”,Power system technology, vol. 28(24),pp.1-4,2004.

[51] ZENG Li-ping, YAO Hong-tao, XIE Xiu-fen, "Fault diagnosis of power transformers based on rule base optimized by genetic algorithm", Journal of Central South University (Science and Technology), vol. 44(3),pp. 1018-1023,2013.

[52] ZHENG Rui-rui, ZHAO Ji-yin, ZHAO Ting-ting, LI Min, "Power Transformer Fault Diagnosis Based on Genetic Support Vector Machine and Gray Artificial Immune Algorithm”,Proceedings of the CSEE, vol. 31(7),pp.56-63,2011.

[53] WANG Gang, YANG Hai-tao, HU Wei-tao, HUANG Hua-ping, LI Ning-yuan, "Predicting method for dissolved gas in transformer oil based on improved genetic algorithm and LS-SVM", high voltage apparatus, vol. 46 (9),pp.11-15,2010.

[54] Liu Zhibin, Jin Lianwen. LATTICESVM-A new method for multi-class Support Vector machines [J], 2008 International Joint Conference on Neural Networks $2008: 727-733$.

[55] DONG Ming, MENG Yuan-yuan, XU Chang-xiang, YAN Zhang, "Fault diagnosis model for power transformer based on support vector machine and dissolved gas analysis”, Proceedings of the CSEE, vol. 23(7),pp.88-92,2003.

[56] [69] RAN WANG , SAM KWONG , DE-GANG CHEN, “A New Method for Multi Class Support Vector Machines by Training Least Number of Classifiers", Proceedings of the 2011 International Conference on Machine Learning and Cybernetics, Guilin, pp.648- 653, 2011.

[57] XIAO Yan-cai, CHEN Xiu-hai, "The improved M-ary support vector machine and its application in power transformer fault diagnosis", Journal of shanghai jiaotong university, vol.42(12),pp.2033-2035,2008.

[58] Barlow R. E., Hunter L. C., "Optimum preventive maintenance policies”, Operations Research, vol. 8(1),pp. 90-100,1960.

[59] J.C. Steed, “Condition monitoring applied to power transformer-an REC experience", Second International Conference on the Reliability of Transmission and Distribution Equipment , vol.(1),pp.109-114,1995.

[60] ZHANG Yi-yi, LIAO Rui-jin,YANG Li-jun,MENG Fan-jin, LI Jin-zhong, Cheng Huan-chao. Maintenance decision making of power transformer by integrated considering reliability and economy [J],Transactions of china electro technical society , vol.29 (11),pp. 192-201,2014.

[61] LIAO Rui-jin, ZHENG Han-bo, YANG Li-jun, ZHANG Yi-yi, HUANG Fei-long, SUN Cai-xin, "A power transformer insulation condition assessment method based on set pair analysis”,Automation of electric power systems, vol.34(21),pp.55-60,2010.

[62] WANG Fu-zhong, WANG Hai-ling, "220 kV transformer condition assessment strategy based on fuzzy theory", Journal of henan polytechnic university(natural science), vol.33(3),pp.349-353,2014.

[63] YUAN Zhi-jian, SUN Cai-xin, LI Ai-hua, LI Jian, DU Lin, LIAO Rui-jin, "Study on condition based maintenance policy of transformer based on fuzzy multiple expert and multiple attribute group decision making”, Automation of electric power systems, vol.28(11),pp.66-70, 2004.

[64] WANG Z, LIU Y, GRIFFIN P J, “A combined ANN and expert system tool for transformer fault diagnosis”, IEEE Transactions on Power Delivery, vol.13(4),pp.1224-1229,1998.

[65] NARESH R, SHARMA V, VASHISTH M, “An integrated neural fuzzy approach for fault diagnosis of transformers”, IEEE Transactions on Power Delivery, vol.23(4),pp.2017-2024,2008.

[66] MOFIZUL I S, WU T, LEDWICH G, “ A novel fuzzy logic approach to transformer fault diagnosis”, IEEE Transactions on Dielectrics and Electrical Insulation, vol.7(2),pp.177-186,2000.

[67] WU W, ISLAM S M, LEDWICH C, “A hybrid intelligent system approach to transformer fault diagnosis”, Proceedings of the Australian Universities Power Engineering Conference, Sydney: [s.n.],pp.43-48,1997. 